\title{
Characterising the Protostellar Population of the Magellanic Clouds with VLT/SINFONI
}

\author{
Jacob Ward ${ }^{1}$, Joana Oliveira ${ }^{1}$, Jacco van Loon ${ }^{1}$, Marta Sewilo ${ }^{2}$ \\ ${ }^{1}$ Faculty of Natural Sciences, Keele University, ST5 5BG, UK \\ email: j.1.ward@keele.ac.uk \\ ${ }^{2}$ Space Science Institute, Boulder, CO 80301, USA
}

\begin{abstract}
.
At distances of $\sim 50 \mathrm{kpc}$ and $\sim 60 \mathrm{kpc}$ for the Large Magellanic Cloud (LMC) and Small Magellanic Cloud (SMC) respectively the Magellanic Clouds present us with a unique opportunity to study star formation in environments outside our own galaxy. Through Spitzer and Herschel photometry and spectroscopy, samples of Young Stellar Objects (YSOs) have been selected and spectroscpically confirmed in the Magellanic Clouds. Here we present some of the key results of our SINFONI $K$-band observations towards massive YSOs in the Magellanic Clouds. We resolve a number of Spitzer sources into multiple, previously unresolved, components and our analysis of emission lines suggest higher accretion rates and different disc properties compared with massive YSOs in the Milky Way.
\end{abstract}

Keywords. stars: formation, Magellanic Clouds, circumstellar matter, infrared: stars

\section{Introduction}

The vast majority of what is known of the star formation process is based on studies of Galactic star forming regions. This presents a relatively limited view of star formation as it neglects how differing galactic properties such as metallicity and large scale tidal forces affect star formation on small scales. The Magellanic Clouds provide a unique opportunity to study star formation in environments outside our own galaxy. At distances of $\sim 50 \mathrm{kpc}$ and $\sim 60 \mathrm{kpc}$ for the LMC and SMC respectively, they allow us to study star formation on both the scale of entire Galaxies and that of individual Young Stellar Objects (YSOs). The relatively low metallicities of the Magellanic Clouds $\left(\mathrm{Z}_{L M C} \sim 0.4 \mathrm{Z}_{\odot}, \mathrm{Z}_{S M C} \sim 0.2 \mathrm{Z}_{\odot}\right)$ also means that they provide us with two intermediate points towards the low metallicity universe. Through the SAGE (Meixner et al. 2006), SAGE-SMC (Gordon et al. 2011) and HERITAGE (Meixner et al. 2010) surveys, Spitzer and Herschel observations have provided a valuable insight into star formation in the Magellanic Clouds but with relatively poor spatial resolution. To study individual YSOs on sub-parsec scales, ground based follow up observations are required using large ground-based telescopes.

Our data set is composed of 20 sources in the SMC selected from Oliveira et al.(2013) with the additions of sources in the $\mathrm{H}$ II regions $\mathrm{N} 81$ and $\mathrm{N} 88$ as well as three sources in the LMC H II region N113 (Ward et al. 2015). Originally selected from the Spitzer SAGE and SAGESMC catalogues, all of our targets have been previously confirmed as YSOs through Spitzer and Herschel spectroscopic observations (Seale et al. 2009; Oliveira et al. 2013). The targets were observed in the K-band over 3 nights in October 2013 using the SINFONI integral field spectrograph at the VLT with a spatial resolution of 0.11 , giving us access to a number of $\mathrm{H}_{2}$ emission lines as well as a $\mathrm{He}$ I emission line and the $\mathrm{H}$ I accretion tracer $\mathrm{Br} \gamma$.

\section{Overview}

We resolve multiple K-band continuum sources in seven out of 23 fields yielding datasets of 6 sources in N113 and 30 in the SMC. 


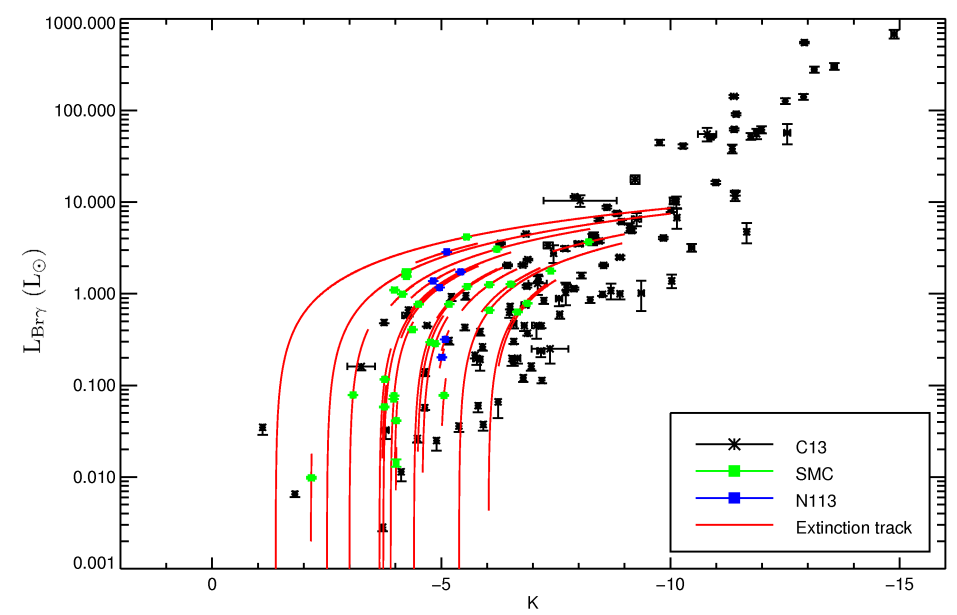

Figure 1. Br $\gamma$ luminosity (in $\mathrm{L}_{\odot}$ versus absolute $K$-band magnitude. Red extinction tracks represent the uncertainty originating from the extinction calculation.

In general our sample exhibits high $\operatorname{Br} \gamma$ luminosities / $K$-band magnitude ratios compared with similar objects in the Milky Way from Cooper et al. (2013), suggestive of higher accretion rates. This would be consistent with the results of De Marchi et al. (2011) and Spezzi et al. (2012), finding that low mass pre-main sequence stars exhibit higher accretion rates in the Magellanic Clouds than in the Milky Way. The effects of metallicity on H I emission are expected to be small (Kudritzki 2002) and we see no excess He I emission compared to the Galactic sample.

Morphologically, two sources in N113 and one in the SMC (N88A) have been classified as compact $\mathrm{H}$ II regions due to significant, extended $\mathrm{Br} \gamma$ and He I emission. Additionally eight extended molecular outflows have been observed, the largest extending to almost $0.5 \mathrm{pc}$ from the continuum source, indicative of early stage YSOs. Velocity gradients have been observed in a number of these structures.

The CO bandhead emission, associated with discs in massive YSOs, is absent in the spectra of all but one of our observed sources. Whilst the detection of the CO bandhead is heavily dependant on the systems geometry and the detection rate in the Galaxy is low $(\sim 17 \%$; Cooper et al. 2013), the lack of all but one weak CO bandhead detection is suggestive of disc properties which differ significantly from Galactic massive YSOs.

\section{References}

Cooper, H.D.B. et al. 2013, MNRAS, 430, 1125

De Marchi, G. et al. 2011, ApJ, 740, 11

Gordon, K.D. et al. 2011, AJ, 142, 102

Kudritzki, R. P. 2002, ApJ, 577, 389

Meixner, M. et al. 2006, AJ, 132, 2268

Meixner, M. et al. 2010, A\&A, 518, L71

Oliveira, J.M. et al. 2013, MNRAS, 428, 3001

Seale, J.P. et al. 2009, ApJ, 699, 150

Spezzi, L. et al 2012, MNRAS, 421, 78

Ward, J.L.; Olivera, J.M.; van Loon, J. Th. \& Sewiło, M. 2015, Accepted in MNRAS 